\title{
COVID-19 vaccine hesitancy among Chinese residents under the free vaccination policy
}

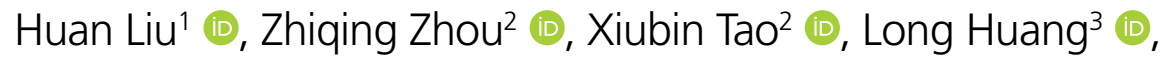 \\ Ergang Zhu ${ }^{4}\left(\mathbb{B}\right.$, Liang $\mathrm{Yu}^{5} \mathbb{1}^{\circ}$, Ming Zhang ${ }^{6 *}$
}

\begin{abstract}
SUMMARY
OBJECTIVE: This study aimed to assess the attitudes of Chinese residents toward COVID-19 vaccines and explore the potential drivers for Chinese residents' vaccine hesitancy.

METHODS: A cross-sectional survey was conducted from February 16 to March 16, 2021, by administering an online questionnaire to the Chinese residents.

RESULTS: Of 5240 residents who completed the survey, 464 (8.9\%) participants reported to have had one shot, and 348 (6.6\%) reported to have had 2 shots. At the time the questionnaire was administered, 2298 (43.9\%) participants reported they wanted to get vaccinated, while 2255 (43.0\%) declared that they still did not know, and 687 (13.1\%) respondents declared vaccine refusal. Overall, 2255 (43\%) participants were categorized as vaccine hesitancy. Female participants $(p=0.000),<20$ years old $(p=0.000)$, have low risk of COVID-19 $(p=0.000)$ infection and strong associations of vaccine hesitancy. eHealth literacy was a protective factor.

CONCLUSIONS: The results of this study show high rates of vaccine hesitancy in China. This could pose a serious threat to the preventive measures that aimed at controlling COVID-19 spread in the country. The government and different media platforms should encourage the dissemination of correct information about vaccines, the communities and medical staff to improve residents' knowledge about vaccines, and strive to improve residents' electronic health literacy.

KEYWORDS: COVID-19. Vaccine. China. Government.
\end{abstract}

\section{INTRODUCTION}

The Coronavirus disease 2019 (COVID-19) was initially reported in Wuhan, Hubei province, China, in December 2019. And due to its rapid worldwide distribution, the World Health Organization (WHO) declared it a pandemic in 2020 ${ }^{1}$. COVID-19 pandemic has brought an unprecedented harm to human health and economic development, from all over the world. The pandemic has infected more than 160 million people and claimed more than 3.3 million lives. Vaccines have been proven to be an extremely effective way of dealing with epidemics in the past. Mathematic modeling indicates that $75 \%$ coverage is needed to reach the herd-immunity threshold to extinguish the ongoing pandemic ${ }^{2}$. However, the vaccine hesitancy has emerged as a major public health problem, topping the list of threats to global health.

Investigations of public attitudes during the pandemic have revealed that details about vaccine and recipient characteristics may influence eventual uptake. To our best knowledge, COVID-19 vaccination acceptance among Chinese residents

\footnotetext{
${ }^{1}$ Yijishan Hospital of Wannan Medical College, Department of Hemodialysis - Anhui, China.

${ }^{2}$ Yijishan Hospital Affiliated to Wannan Medical College, Department of Nursing - Anhui, China.

${ }^{3}$ Wannan Medical College, School of Humanities and Management - Anhui, China.

${ }^{4}$ Wannan Medical College, School of Comprehensive Foundation - Anhui, China.

${ }^{5}$ Wannan Medical College, School of Humanities and Management - Anhui, China.

${ }^{6}$ Wannan Medical College, School of Innovation and Entrepreneurship - Anhui, China.

*Corresponding author: wnyxyzhangming@foxmail.com

Conflicts of interest: the authors declare there are no conflicts of interest. Funding: none.

Received on July 13, 2021. Accepted on August 10, 2021.
} 
has not been studied. The purpose of this study was to evaluate the vaccination acceptance of Chinese residents.

\section{METHODS}

\section{Study design and setting}

A cross-sectional survey was conducted from February 16 to March 16, 2021, by administering an online questionnaire to the Chinese residents. The participants in this study were recruited from an online survey, via a self-reported questionnaire, using Sojump (https://www.wjx.cn/). All the participants were recruited using simplified-snowball sampling technique, and the invited participants were asked to pass the invitation to their QQ and WeChat contacts. Before starting the survey, all participants had to give their informed consent, and the purpose of the study must be explained. Participants could withdraw from the survey at any time. All information and opinions provided by participants were anonymous and confidential.

\section{Questionnaire development}

The questionnaire was divided into two sections:

- Demographic information;

- eHealth Literacy Scale.

\section{Assessment and evaluation}

\section{Demographic information}

This section collects information about the general characteristics of the survey respondents, including gender, age, marital status, education level, if they had existing chronic diseases or not, risk of infection COVID-19, etc.

\subsection{2. eHealth Literacy Scale (eHEALS).}

The eHealth Literacy Scale (eHEALS) was compiled in 2006 by Norman and Skinner ${ }^{3}$. Guo Shuaijun et al. chineseized and revised the scale in $2014^{4}$. There are a total of eight items, including application, evaluation, and decision-making. The Likert 5-level scoring method is used. From "very inconsistent" to "very consistent", 1-5 points are counted, and the total score is $8-40$ points. A score of $\geq 32$ is considered as qualified for electronic health literacy, and a score of less than 32 is considered as unqualified for electronic health. Cronbach's alpha coefficient of eHEALS was 0.975 .

\section{Statistical analysis}

Information was collected from Sojump. All data were analyzed by IBM SPSS version 22.0 (SPSS Inc., Chicago, IL,
USA). Frequencies and percentages were performed for categorical data. The chi-square test was used to verify the differences of categorical variables between groups. Binary logistic regression analyses were used to explore the impact factor of anti-COVID-19 vaccine hesitancy. The test level was $a=0.05$, that is, a p-value of less than 0.05 was considered statistically significant. Odds ratios (ORs) and 95\% confidence intervals (CIs) were used to estimate associations.

\section{RESULTS}

\section{Demographic characteristics}

A total of 5240 participants were included in this investigation. The average age of the 5240 participants was 25.07 years $(\mathrm{SD}=9.655$; range $10-70$ ), of which $19.8 \%$ were men; $44.2 \%$ of the participants lived in the city. Among all residents participating in the survey, 294 (5.6\%) residents were high school graduate or less, 2779 (53\%) residents were junior college, 1766 (33.7\%) residents were college graduate, and 401 (7.7\%) residents were advanced degree. Demographic data of the study samples are presented in Table 1.

\section{The level of the COVID-19 vaccine hesitancy}

A total of $464(8.9 \%)$ participants reported to have had 1 shot, and 348(6.6\%) participants reported to have had two shots. At the time the questionnaire was administered, 2298 (43.9\%) participants reported they wanted to get vaccinated, while 2255 (43.0\%) participants declared that they still did not know, and 687 (13.1\%) participants declared vaccine refusal. Overall, 2255 (43\%) participants were categorized as vaccine hesitancy.

\section{Associated factors of COVID-19 vaccine hesitancy}

When comparing the COVID-19 vaccine hesitancy and not, a significant difference was found in six items, such as gender, age, education, risk of infection COVID-19, chronic diseases, and eHealth Literacy (Table 1).

\section{Regression analyses for COVID-19 vaccine hesitancy}

To predict whether the independent variables were associated with COVID-19 Vaccine hesitancy, we used the logistic regression analyses. As shown in Table 2, four variables were found to be associated with COVID-19 Vaccine hesitancy, such as gender $(\mathrm{p}<0.01)$, age $(\mathrm{p}<0.01)$, risk of infection COVID-19 $(\mathrm{p}<0.01)$, and eHealth Literacy $(\mathrm{p}<0.01)$. 
Table 1. Respondent characteristics $(n=5240)$.

\begin{tabular}{|c|c|c|c|c|c|c|}
\hline & \multirow{2}{*}{ Characteristic } & \multirow{2}{*}{ Total n (\%) } & \multicolumn{2}{|c|}{ Hesitate n (\%) } & \multirow{2}{*}{$\chi^{2}$} & \multirow{2}{*}{ p-value } \\
\hline & & & No & Yes & & \\
\hline \multirow{2}{*}{ Gender } & Male & $1037(19.8)$ & $689(66.4)$ & $348(33.6)$ & \multirow{2}{*}{47.356} & \multirow{2}{*}{$0.000 * *$} \\
\hline & Female & $4203(80.2)$ & $2296(54.6)$ & $1907(45.4)$ & & \\
\hline \multirow{4}{*}{ Age } & $<20$ & 1994(38.1) & $832(41.7)$ & $1162(58.3)$ & \multirow{4}{*}{431.112} & \multirow{4}{*}{$0.000 * *$} \\
\hline & $20-30$ & 1884(36) & 1095(58.1) & 789(41.9) & & \\
\hline & $30-50$ & $1196(22.7)$ & $939(78.5)$ & $257(21.5)$ & & \\
\hline & $>50$ & $166(3.2)$ & $119(71.7)$ & $47(28.3)$ & & \\
\hline \multirow{4}{*}{ Education } & High school graduate or less & 294(5.6) & $170(57.8)$ & $124(42.2)$ & \multirow{4}{*}{173.738} & \multirow{4}{*}{$0.000 * *$} \\
\hline & Junior college & 2779(53) & $1354(48.7)$ & $1425(51.3)$ & & \\
\hline & College graduate & 1766(33.7) & $1190(67.4)$ & $576(32.6)$ & & \\
\hline & Advanced degree & $401(7.7)$ & $271(67.6)$ & $130(32.4)$ & & \\
\hline \multirow{4}{*}{$\begin{array}{l}\text { Your risk of } \\
\text { infection } \\
\text { COVID-19 }\end{array}$} & Low & $3159(60.3)$ & $1559(49.4)$ & $1600(50.6)$ & \multirow{4}{*}{323.685} & \multirow{4}{*}{$0.000 * *$} \\
\hline & General & $1141(21.8)$ & $651(57.1)$ & $490(42.9)$ & & \\
\hline & High & $940(17.9)$ & $775(82.4)$ & $165(17.6)$ & & \\
\hline & Other & 206(3.9) & $68(33)$ & $138(67)$ & & \\
\hline \multirow{2}{*}{$\begin{array}{l}\text { Chronic } \\
\text { diseases }\end{array}$} & No & 4799(91.6) & $2714(56.6)$ & 2085(43.4) & \multirow{2}{*}{3.952} & \multirow{2}{*}{$0.047^{*}$} \\
\hline & Yes & $441(8.4)$ & 271(61.5) & $170(38.5)$ & & \\
\hline \multirow{2}{*}{$\begin{array}{l}\text { eHealth } \\
\text { Literacy }\end{array}$} & No & $2365(45.1)$ & $1107(46.8)$ & $1258(53.2)$ & \multirow{2}{*}{181.433} & \multirow{2}{*}{0.000 ** } \\
\hline & Yes & $2875(54.9)$ & $1878(65.3)$ & $997(34.7)$ & & \\
\hline
\end{tabular}

${ }^{*} p<0.05 ; * * p<0.01$.

Table 2. Logistic regression analyses for COVID-19 vaccine hesitancy ( $N=5240)$.

\begin{tabular}{|c|c|c|c|c|c|c|c|}
\hline & \multirow[b]{2}{*}{ B } & \multirow[b]{2}{*}{ SE } & \multirow[b]{2}{*}{ Wals } & \multirow[b]{2}{*}{ Sig. } & \multirow[b]{2}{*}{$\operatorname{Exp}(B)$} & \multicolumn{2}{|c|}{$95 \% \mathrm{Cl}$} \\
\hline & & & & & & $\begin{array}{l}\text { Lower } \\
\text { bound }\end{array}$ & $\begin{array}{l}\text { Upper } \\
\text { bound }\end{array}$ \\
\hline Gender & 0.331 & 0.079 & 17.358 & 0.000 & 1.392 & 1.191 & 1.626 \\
\hline Age $<20$ & & & 22.259 & 0.000 & & & \\
\hline $20-30$ & -0.311 & 0.071 & 18.962 & 0.000 & 0.733 & 0.637 & 0.843 \\
\hline $30-50$ & -0.477 & 0.152 & 9.808 & 0.002 & 0.621 & 0.461 & 0.837 \\
\hline$>50$ & -0.281 & 0.23 & 1.495 & 0.221 & 0.755 & 0.481 & 1.185 \\
\hline Your risk of infection COVID-19 (Low) & & & 72.398 & 0.000 & & & \\
\hline General & -0.132 & 0.077 & 2.974 & 0.085 & 0.876 & 0.754 & 1.018 \\
\hline High & -0.898 & 0.106 & 72.136 & 0.000 & 0.407 & 0.331 & 0.501 \\
\hline eHealth Literacy (No) & -0.353 & 0.065 & 29.732 & 0.000 & 0.702 & 0.618 & 0.797 \\
\hline Constant & 0.631 & 0.095 & 44.244 & 0.000 & 1.88 & & \\
\hline
\end{tabular}

$F=22.274 ; p=0.000$, Cox Snell $R^{2}=0.161$, Nagelkerke $R^{2}=0.216$. ${ }^{*} p<0.05,{ }^{*} p<0.01$. 


\section{DISCUSSION}

\section{Key findings}

This study found that three months after the vaccine was launched, among 5240 Chinese residents, only $43.9 \%$, $(n=2298)$ reported to be willing to accept COVID-19 vaccination. About 43\% ( $\mathrm{n}=2255)$ of the participants indicated unsure and $13.1 \%(\mathrm{n}=687)$ indicated that they would refuse the vaccination. A total of 2255 (43\%) participants were categorized as vaccine hesitancy; the overall hesitation rate was high. During the data collection period, February 16 to March 16, 2021, the total number of confirmed COVID-19 cases in China every day is almost zero, and the confirmed cases are all imported cases from abroad. On the other hand, various false information circulating on Internet and social media is also a determining factor influencing vaccine hesitancy in some groups.

It is worth noting that our data represent a high level of hesitation rate of the COVID-19 vaccine among the study population. Although China has successfully contained the epidemic, it should not underestimate the possibility of another outbreak. Previous studies have shown that high perceived susceptibility and high risk perception can be translated into better preventive measures and are related to enhanced epidemic control capabilities 5 . Therefore, sustainable prevention and control measures should be encouraged.

\section{Differences in vaccine hesitancy}

In our study, women were associated with higher rates of COVID19 vaccine hesitancy, which is consistent with the previous results ${ }^{6}$. One possible explanation is that women are more likely to fear the unknown ${ }^{7}$, and more cautious about trying new things. Some false claims about COVID-19 vaccines were believed that the vaccine is not good to female health ${ }^{8}$. And females are less educated, and the main source of information is social media platforms rather than medical institutions and doctors platforms.

In our study, $58.3 \%$ of the participants under 20 years old reported hesitancy to receive the COVID-19 vaccine. People under the age of 20 have the highest hesitation rate. In this study, the subjects under the age of 20 are basically college students. Because of the centralized management of the school, they feel more secure, so they show more hesitation. Young people feel healthier and, therefore, show more vaccine hesitancy?.

Risk perception is crucial for vaccine decision-making. The present study found that perceived risk of contracting COVID19 was negatively associated with hesitation to have a COVID-19 vaccination. Although the spread of the virus was well controlled after The Chinese government implementing a series of actions, the Chinese people experienced the earliest threats concerning COVID-19. The prevalence rates of symptoms of psychological distress were relatively high in the general population during the early stages of the COVID-19 pandemic in China ${ }^{10}$. These negative mood experiences will become the motivation for individuals to vaccinate against COVID-19.

These findings demonstrate that the trust in vaccines and government is key. In particular, addressing patients' concerns about the side effects through open and transparent communication may be very useful in building trust and confidence ${ }^{11}$. The huge amount of false/disinformation spread on social media casts a shadow on people's understanding of COVID-19 vaccine cause great confusion among the public.

The present study found that people with chronic diseases have a lower rate of vaccine hesitancy as others. Evidence suggests that patients with chronic diseases are particularly prone to serious complications and mortality than healthy individuals ${ }^{12}$, which can lead to increased willingness to vaccinate.

Today, eHealth literacy is defined as "the ability to search, find, understand, and evaluate health information from electronic resources and apply the acquired knowledge to address or solve health problems"13. The internet has always been a major source of COVID-19 information, especially during the period of lockdown. However, many online information about COVID-19 lacks scientific rigor ${ }^{14}$. Theoretically, people with higher eHL should have the ability to efficiently process the flood of health-related information on the Internet and showed less anxiety and hesitation when faced with false information about COVID-19 vaccines on the Internet.

\section{CONCLUSIONS}

The results of this study show high rates of vaccine hesitancy in China. This could pose a serious threat to the preventive measures that aimed at controlling COVID-19 spread in the country. The government and different media platforms should encourage the dissemination of correct information about vaccines, the communities, and medical staff to improve residents' knowledge about vaccines and strive to improve residents' electronic health literacy. Our study further show that government provided timely information (such as side effects of the vaccine) by social media, and adequate protective supplies might mitigate the level of the hesitation of the COVID-19 vaccine.

\section{AUTHORS" CONTRIBUTIONS}

HL: Conceptualization, Methodology, Software, Validation, Writing-original draft preparation. MZ: Conceptualization, Validation, Resources, Writing-original draft preparation. ZZ: Software. XT: Validation. LH: Formal analysis. EZ: Investigation. LY: data curation. 


\section{REFERENCES}

1. Fisher D, Teo YY, Nabarro D. Assessing national performance in response to COVID-19. Lancet. 2020;396(10252):653-5. https://doi.org/10.1016/S0140-6736(20)31601-9

2. Zhang KC, Fang $Y, \mathrm{Cao} H$, Chen $\mathrm{H}, \mathrm{Hu} T$, Chen $Y Q$, et al. Parental acceptability of COVID-19 vaccination for children under the age of 18 years: cross-sectional online survey. JMIR Pediatr Parent. 2020;3(2):e24827. https://doi. org/10.2196/24827

3. Norman CD, Skinner HA. eHealth Literacy: Essential Skills for Consumer Health in a Networked World. J Med Internet Res. 2006;8(2):e9. https://doi.org/10.2196/jmir.8.2.e9

4. Guo SJ, Yu XM, Sun YY, Nie D, Li X, Wang L, et al. Adaptation and evaluation of Chinese version of eHEALS and its usage among senior high school students. Chin J Health Educ. 2013;29(2):106-8. https://doi.org/10.16168/j.cnki.issn.10029982.2013.02.019

5. Verelst F, Willem L, Beutels P. Behavioural change models for infectious disease transmission: a systematic review (20102015). J R Soc Interface. 2016;13(125):20160820. https:// doi.org/10.1098/rsif.2016.0820

6. Batty GD, Deary IJ, Fawns-Ritchie C, Gale CR, Altschul D. Pre-pandemic Cognitive Function and COVID-19 Vaccine Hesitancy: Cohort Study. Brain Behav Immun. 2021;96:100-5. https://doi.org/10.1016/j.bbi.2021.05.016

7. McLean CP, Anderson ER. Brave men and timid women? A review of the gender differences in fear and anxiety. Clin Psychol Rev. 2009;29(6):496-505. https://doi.org/10.1016/j. cpr.2009.05.003
8. Male V. Are COVID-19 vaccines safe in pregnancy? Nat Rev Immunol. 2021;21(4):268. https://doi.org/10.1038/s41577021-00533-y

9. Dror AA, Eisenbach N, Taiber S, Morozov NG, Mizrachi M, Zigron $A$, et al. Vaccine hesitancy: the next challenge in the fight against COVID-19. Eur J Epidemiol. 2020;35(8):775-9. https://doi.org/10.1007/s10654-020-00671-y

10. Ding $Y, X u J$, Huang $S$, Li P, Lu C, Xie S. Risk perception and depression in public health crises: evidence from the COVID-19 crisis in China. Int J Environ Res Public Health. 2020;17(16):5728. https://doi.org/10.3390/ijerph17165728

11. Larson HJ, Figueiredo $A$, Xiahong $Z$, Schulz WS, Verger $P$, Johnston IG, et al. The state of vaccine confidence 2016: global insights through a 67-Country Survey. EBioMedicine. 2016;12:295-301. https://doi.org/10.1016/j.ebiom.2016.08.042

12. Schwarze J, Openshaw $P$, Jha A, Del Giacco SR, Firinu D, Tsilochristou $O$, et al. Influenza burden, prevention, and treatment in asthma-A scoping review by the EAACI Influenza in asthma task force. Allergy. 2018;73(6):1151-81. https:// doi.org/10.1111/all.13333

13. Norman CD, Skinner HA. eHEALS: The eHealth Literacy Scale. J Med Internet Res. 2006;8(4):e27. https://doi.org/10.2196/ jmir.8.4.e27

14. Fan KS, Ghani SA, Machairas N, Lenti L, Fan KH, Richardson $D$, et al. COVID-19 prevention and treatment information on the internet: a systematic analysis and quality assessment. BMJ Open. 2020;10(9):e040487. https://doi.org/10.1136/ bmjopen-2020-040487 